\title{
Medical Image of the Week: Superior Vena Cava Syndrome
}

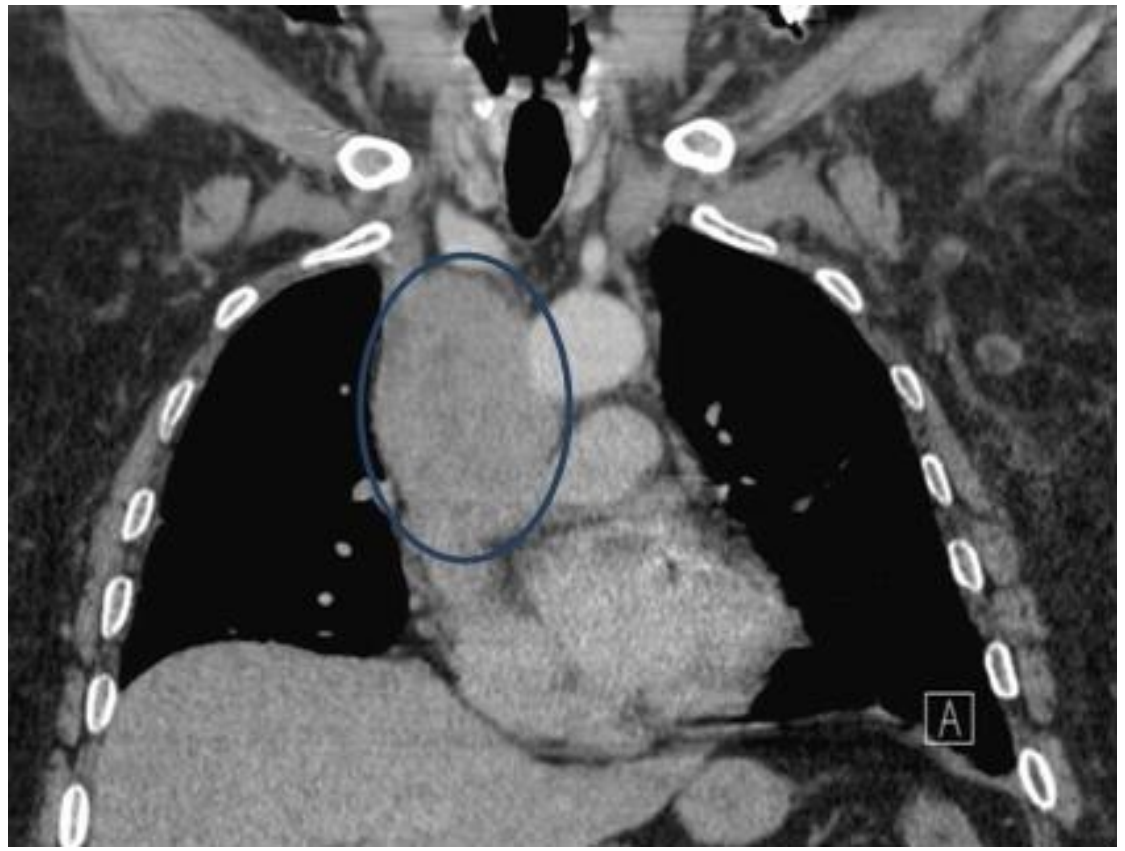

Figure 1. Contrast-enhanced CT of the chest in the arterial phase in the coronal plane demonstrates a large paratracheal mass (blue circle) that is invading the SVC resulting in the tumor thrombus noted in right heart chambers.

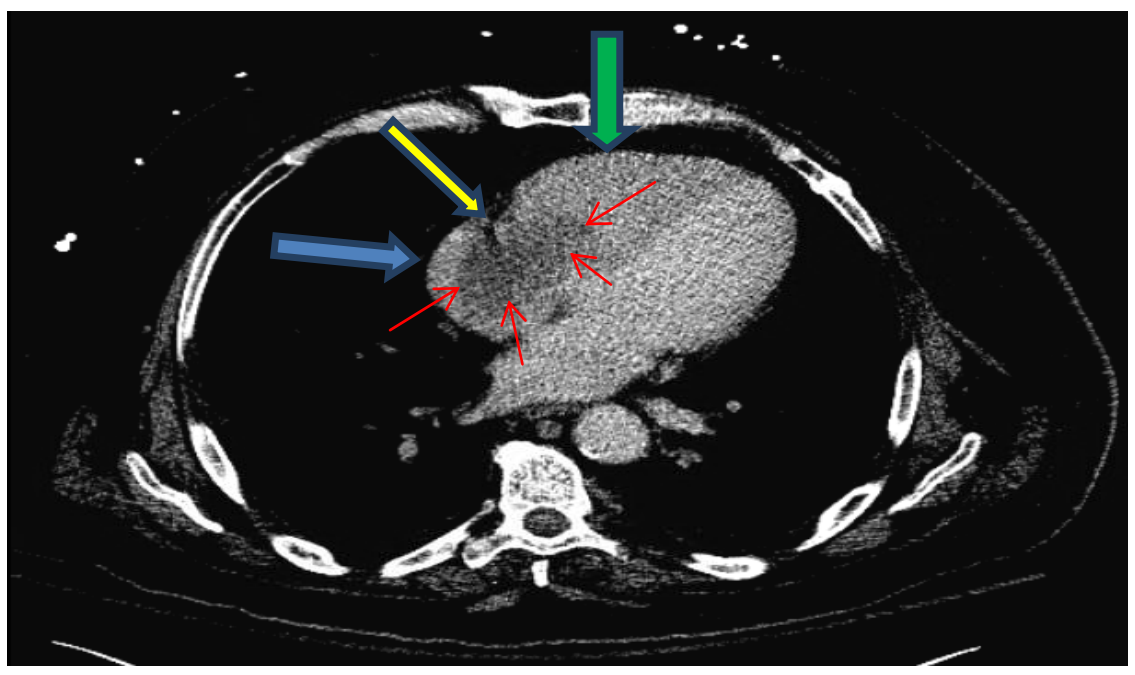

Figure 2. Contrast-enhanced CT of the chest in the arterial phase at the level of the right atrium (blue arrow), tricuspid annulus (yellow arrow), and right ventricle (green arrow) demonstrates a thrombus extending from the right atrium across the tricuspid valve in to the right ventricle. 
A 65 year old Native American man with past medical history significant for hypertension presented with a two week history of generalized edema, most prominent in the face and upper extremities. The patient had gained $30 \mathrm{lbs}$ in the previous 6 months. He denied any fever, night sweats, dyspnea, hemoptysis, change in voice, chest pain, abdominal pain, nausea, vomiting, or hematemesis but did acknowledge a 40+ pack-year smoking history. Family history was significant for two brothers deceased from lung cancer. On presentation, he was hemodynamically stable, had visibly distended neck veins and collateral veins on the chest and abdomen. Routine laboratory tests included a comprehensive metabolic panel remarkable for mild transaminitis, complete blood count with thrombocytopenia $(69,000)$ and mild anemia (hemoglobin 13.5). Urinalysis and infectious workup were unremarkable. A CT chest/abdomen/pelvis confirmed superior vena cava (SVC) syndrome from a thrombus in the right atrium extending cephalad into the SVC and left brachiocephalic vein. Patient was started on dexamethasone $4 \mathrm{mg}$ every 6 hours and a heparin drip. A fine needle biopsy of the large mediastinal paratracheal mass showed non-small cell lung carcinoma. He received cycle 1 of carboplatin and docetaxel. Five days after chemotherapy, patient had large volume hemoptysis. Repeat CTA chest demonstrated enlargement of the right suprahilar mass invading the mediastinum/SVC with extension into the right atrium and crossing into the right ventricle (Image 1 and 2). Considering severity of the disease and poor prognosis patient and patient's family accepted comfort care.

SVC syndrome results from mechanical obstruction of the SVC. Dyspnea, facial swelling and distended neck veins are the characteristic clinical manifestations (1). In the era of antibiotics, $70-90 \%$ of cases are due to mediastinal malignancies (2). Symptomatic relief with steroids, radiation/chemotherapy and intravascular stents are mainstays of emergent treatment (1). However, similar to our case, due to aggressive nature of the disease the mortality is inevitable.

Manjinder Kaur DO, Charity Adusei MS III, Tammer Elaini MD, and Laura Meinke MD Department of Medicine

The University of Arizona and Sourthern Arizona VA Health Care System

Tucson, AZ, USA

\section{References}

1. Khan UA, Shanholtz CB, McCurdy MT. Oncologic mechanical emergencies. Emerg Med Clin North Am. 2014;32(3):495-508. [CrossRef] [PubMed]

2. Rossow CF, Luks AM. A 68-year-old woman with hoarseness and upper airway edema. Ann Am Thorac Soc. 2014;11(4):668-70. [CrossRef] [PubMed] 